\title{
Calculations of solid solubility in supercritical fluids using a simplified cluster solvation model
}

\author{
Kong-Wei Cheng ${ }^{1}$, Muoi Tang ${ }^{2}$, Yan-Ping Chen* \\ Department of Chemical Engineering, National Taiwan University, Taipei, Taiwan, ROC
}

Received 20 March 2003; accepted 20 June 2003

\begin{abstract}
A simplified cluster solvation model was employed in this study to correlate the solid solubility in supercritical fluids. Based on the concept of cluster solvation, several supercritical molecules were associated with a solid solute in chemical equilibrium. Solid solubility was calculated by applying the equilibrium criteria for the cluster formation process. This simplified model has two temperature-independent binary parameters. It is observed that the overall deviation in solid solubility calculations of binary systems from this model is comparable to that from other semi-empirical models with more optimally fitted parameters. This simplified cluster solvation model was extended to ternary and multi-component calculations by directly using the parameters from binary correlation results. Without any additional parameter, the predicted accuracy is still acceptable. Application of this model to systems with co-solvent has also been examined. A general trend for the variation of the effective association number with co-solvent concentration is observed.
\end{abstract}

(C) 2003 Elsevier B.V. All rights reserved.

Keywords: Method of calculation; Solid-fluid equilibria; Mixture

\section{Introduction}

The application of supercritical fluid (SCF) in modern technology of extraction, purification, reaction, and crystal growth has attracted more and more attention in recent years [1]. One key point for designing a supercritical process is the equilibrium solubility data. Besides accurate measurements of high-pressure phase equilibrium data, suitable thermodynamic models are highly required to correlate and predict the equilibrium solid solubility in supercritical fluids.

The solid solubility in supercritical fluid can be calculated by equation of state (EOS) method, activity coefficient models, semi-empirical equations or chemical equilibrium approach. EOS method with

\footnotetext{
* Corresponding author. Fax: +886-2-2362-3040.

E-mail address: ypchen@ ccms.ntu.edu.tw (Y.-P. Chen).

${ }^{1}$ Present address: Advanced Energy Technology Lab., Energy \& Resource Laboratories, ITRI, Hsinchu, Taiwan, ROC.

${ }^{2}$ Present address: Department of Chemical Engineering, Chinese Culture University, Taipei, Taiwan, ROC.
} 
traditional van der Waals (VDW) mixing rules is usually employed to calculate the solid solubility in supercritical fluids [2]. The uncertain solid properties (e.g. critical constants and vapor pressure) and other empirical parameters in the EOS method limit its use for predictive or multi-component computations $[3,4]$. An alternative method without using critical constants or solid vapor pressure is the application of solution model [5,6]. However, no attempt in applying solution model to multi-component systems has yet been presented. In the semi-empirical equation approach, Mendez-Santiago and Teja [7] proposed an accurate simplified equation based on the dilution solution theory. There are three parameters for each binary system. The number of parameters can be reduced to two if the sublimation pressure of solid is available. Their model has been extended to ternary systems with co-solvents [8].

To develop an improved calculation model, approximation of the actual phenomena of solute in the supercritical phase needs to be examined. Recently, the molecular interaction at high pressure has been studied. These investigations reported the formation of clusters, or aggregates of the solvent molecules around the solute, at high-pressure conditions [9-13]. Brennecke and Eckert [14] used spectroscopic technology to study cluster phenomena in supercritical phase and presented an evidence for the formation of dimers. These results suggest that the formation of aggregates through chemical equilibrium should be considered in solid solubility calculation. Thermodynamic models based on the cluster solvation concept $[15,16]$ were applied for correlating the solubility of solid in supercritical fluid. These methods show satisfactory results on binary systems. Parameters in these models, however, are either temperature-dependent or without generalization to multi-component mixtures.

In this study, a simplified model based on the cluster solvation concept is derived for correlating the solid solubility in various supercritical fluids. Calculation results and the best-fitted binary parameters are presented. This simplified model is further extended for solid solubility calculations of ternary mixtures, and for systems with co-solvents. Comparisons of this model with other approaches are also presented.

\section{Solid solubility from the theory of cluster formation}

A simplified cluster solvation model is employed in this study with the following assumptions. (1) Solvent molecules, free solute molecules, and solvent-solute clusters are at chemical equilibrium in the supercritical fluid phase. (2) A cluster consists of one solute associated with several solvent molecules. The number of associated solvent molecule is the association number $k$. To simplify a practical correlation of experimental solubility data, the average value of $k$ in a certain temperature and pressure range is assumed as a constant. (3) The supercritical fluid is taken as in the vapor phase.

Following these assumptions, an equilibrium equation exists in the SCF-solid system at fixed temperature and pressure (as described in [15]):

$$
\mathrm{S}+k \mathrm{~F} \leftrightarrow \mathrm{SF}_{k}
$$

where $\mathrm{S}$ denotes the solute molecule in the solid phase, $\mathrm{F}$ the bulk solvent, $k$ the association number, and $\mathrm{SF}_{k}$ the cluster. This equation represents the sublimation of the solid molecule into the supercritical phase, and the chemical equilibrium for the cluster formation process. In reality, free solutes, supercritical fluid and solute cluster may co-exist in the supercritical phase. For the simplicity of calculation, we model that all solutes are solvated with an average association number $k$. In a small experimental temperature range of $20-30 \mathrm{~K}$, this average association number is considered as a constant for each binary system. Since it is an average value of all possible clusters with different association numbers and even free solutes, $k$ 
may not be an integer and is allowed to be less than unity. Considering a chemical reaction equilibrium condition, we have:

$$
\sum v_{i} \bar{G}_{i}\left(T, P, z_{i}\right)=0
$$

The partial molar Gibbs energy for species $i$ is written as:

$$
\bar{G}_{i}\left(T, P, z_{i}\right)=\bar{G}_{i}^{\circ}\left(T, P^{\circ}, z_{i}^{\circ}\right)+\left[\bar{G}_{i}\left(T, P, z_{i}\right)-\bar{G}_{i}^{\circ}\left(T, P^{\circ}, z_{i}^{\circ}\right)\right]
$$

where $\bar{G}_{i}^{\circ}$ denotes the partial molar Gibbs energy of the standard state at $T$ and $P^{\circ}$, and $z$ is the mole fraction in the supercritical phase. In this study, the pure component at temperature $T$ and the critical pressure of the supercritical fluid $P_{c, \text { scf }}$ is chosen as the standard state. The partial molar Gibbs energy of each species $i$ is written as:

supercritical fluid : $\quad \bar{G}_{\mathrm{F}}\left(T, P, z_{\mathrm{F}}\right)=G_{\mathrm{F}}^{\circ}\left(T, P_{\mathrm{c}, \mathrm{scf}}\right)+R T \ln \frac{\hat{f}_{\mathrm{F}}\left(T, P, z_{\mathrm{F}}\right)}{f_{\mathrm{F}}\left(T, P_{\mathrm{c}, \mathrm{scf}}\right)}$

solid solute : $\quad \underline{G}_{\mathrm{s}}(T, P)=G_{\mathrm{s}}^{\circ}\left(T, P_{\mathrm{c}, \mathrm{scf}}\right)+R T \ln \frac{f_{\mathrm{s}}(T, P)}{f_{\mathrm{s}}\left(T, P_{\mathrm{c}, \mathrm{scf}}\right)}$

the cluster : $\quad \bar{G}_{\mathrm{SF}_{k}}\left(T, P, z_{\mathrm{SF}_{k}}\right)=G_{\mathrm{SF}_{k}}^{\circ}\left(T, P_{\mathrm{c}, \mathrm{scf}}\right)+R T \ln \frac{\hat{f}_{\mathrm{SF}_{k}}\left(T, P, z_{\mathrm{SF}_{k}}\right)}{f_{\mathrm{SF}_{k}}\left(T, P_{\mathrm{c}, \mathrm{scf}}\right)}$

where $G_{\mathrm{SF}_{k}}^{\circ}$ is the Gibbs energy of the pure cluster, and $G_{\mathrm{F}}^{\circ}$ or $G_{\mathrm{s}}^{\circ}$ the Gibbs energy of pure supercritical fluid or solid solute, respectively. $f_{\mathrm{SF}_{k}}$ is the fugacity of the cluster, and $f_{\mathrm{s}}$ or $f_{\mathrm{F}}$ the fugacity of the solute or the supercritical fluid, respectively.

Applying the conventional expressions for fugacity in a fluid mixture, we have:

$$
\begin{aligned}
& \bar{G}_{\mathrm{F}}\left(T, P, z_{\mathrm{F}}\right)=G_{\mathrm{F}}^{\circ}\left(T, P_{\mathrm{c}, \mathrm{scf}}\right)+R T \ln \frac{z_{\mathrm{F}} \hat{\phi}_{\mathrm{F}}\left(T, P, z_{\mathrm{F}}\right) P}{\phi_{\mathrm{F}}\left(T, P_{\mathrm{c}, \mathrm{scf}}\right) P_{\mathrm{c}, \mathrm{scf}}} \\
& \underline{G}_{\mathrm{s}}(T, P)=G_{\mathrm{s}}^{\circ}\left(T, P_{\mathrm{c}, \mathrm{scf}}\right)+V_{\mathrm{s}}\left(P-P_{\mathrm{c}, \mathrm{scf}}\right) \\
& \bar{G}_{\mathrm{SF}_{k}}\left(T, P, z_{\mathrm{SF}_{k}}\right)=G_{\mathrm{SF}_{k}}^{\circ}\left(T, P_{\mathrm{c}, \mathrm{scf}}\right)+R T \ln \frac{z_{\mathrm{SF}_{k}} \hat{\phi}_{\mathrm{SF}_{k}}\left(T, P, z_{\mathrm{SF}_{k}}\right) P}{\phi_{\mathrm{SF}_{k}}\left(T, P_{\mathrm{c}, \mathrm{scf}}\right) P_{\mathrm{c}, \mathrm{scf}}}
\end{aligned}
$$

Substituting these expressions for partial Gibbs energy into Eq. (2) leads to:

$$
\begin{aligned}
\ln \frac{z_{\mathrm{SF}_{k}}}{z_{\mathrm{F}}^{k}}= & k \ln \frac{\hat{\phi}_{\mathrm{F}}\left(T, P, z_{\mathrm{F}}\right) P}{\phi_{\mathrm{F}}\left(T, P_{\mathrm{c}, \mathrm{scf}}\right) P_{\mathrm{c}, \mathrm{scf}}}+\frac{V_{\mathrm{s}}\left(P-P_{\mathrm{c}, \mathrm{scf}}\right)}{R T}-\ln \frac{\hat{\phi}_{\mathrm{SF}_{k}}\left(T, P, z_{\mathrm{SF}_{k}}\right) P}{\phi_{\mathrm{SF}_{k}}\left(T, P_{\mathrm{c}, \mathrm{scf}}\right) P_{\mathrm{c}, \mathrm{scf}}} \\
& -\frac{\Delta G^{\mathrm{rxn}}\left(T, P_{\mathrm{c}, \mathrm{scf}}\right)}{R T}
\end{aligned}
$$

where the Gibbs energy change due to the cluster formation reaction is:

$$
\Delta G^{\mathrm{rxn}}\left(T, P_{\mathrm{c}, \mathrm{scf}}\right)=G_{\mathrm{SF}_{k}}^{\circ}\left(T, P_{\mathrm{c}, \mathrm{scf}}\right)-k G_{\mathrm{F}}^{\circ}\left(T, P_{\mathrm{c}, \mathrm{scf}}\right)-G_{\mathrm{s}}^{\circ}\left(T, P_{\mathrm{c}, \mathrm{scf}}\right)
$$

There are two parameters $k$ and $\Delta G^{\mathrm{rxn}}$ in this model that are regressed using the experimental solid solubility data. The average association number $k$ is assumed as a constant for each binary system. The 
Gibbs energy change for cluster formation includes enthalpy and entropy contributions, and it is taken as a simple temperature-dependent function in this study. In this simplified model, we have $z_{\mathrm{F}}+z_{\mathrm{SF}_{k}}=1$. The denominator of the left hand side of Eq. (10), $z_{\mathrm{F}}=1-z_{\mathrm{SF}_{k}}$, is further written as a Taylor series expansion. For a small solid solubility usually $<10^{-2}$ or $10^{-3}$ mole fraction, the truncated result gives:

$$
\begin{aligned}
\ln \frac{z_{\mathrm{SF}_{k}}}{\left(1-k z_{\mathrm{SF}_{k}}\right)}= & k \ln \frac{\hat{\phi}_{\mathrm{F}}\left(T, P, z_{\mathrm{F}}\right) P}{\phi_{\mathrm{F}}\left(T, P_{\mathrm{c}, \mathrm{scf}}\right) P_{\mathrm{c}, \mathrm{scf}}}+\frac{V_{\mathrm{s}}\left(P-P_{\mathrm{c}, \mathrm{scf}}\right)}{R T}-\ln \frac{\hat{\phi}_{\mathrm{SF}_{k}}\left(T, P, z_{\mathrm{SF}_{k}}\right) P}{\phi_{\mathrm{SF}_{k}}\left(T, P_{\mathrm{c}, \mathrm{scf}}\right) P_{\mathrm{c}, \mathrm{scf}}} \\
& -\frac{\Delta G^{\mathrm{rxn}}\left(T, P_{\mathrm{c}, \mathrm{scf}}\right)}{R T}
\end{aligned}
$$

The experimental solubility of the solid solute in the supercritical fluid, $y$, is then related to the mole fraction of cluster by [15]:

$$
y=\frac{z_{\mathrm{SF}_{k}}}{\left(1+k z_{\mathrm{SF}_{k}}\right)}
$$

The solute solubility $y$ in a supercritical fluid is calculated using Eqs. (12) and (13). The fugacity coefficients, $\hat{\phi}_{\mathrm{SF}_{k}}$ and $\hat{\phi}_{\mathrm{F}}$, for cluster and supercritical fluid, respectively, are calculated using the Peng-Robinson EOS [17]:

$$
\begin{aligned}
& P=\frac{R T}{V-b}-\frac{a}{V^{2}+2 b V-b^{2}} \\
& a(T)=0.45724\left(\frac{R^{2} T_{\mathrm{c}}^{2}}{P_{\mathrm{c}}}\right) \alpha(T) \\
& \alpha(T)=\left[1+m\left(1-T_{\mathrm{r}}^{0.5}\right)\right]^{2} \\
& m=0.37464+1.54226 \omega-0.26992 \omega^{2} \\
& b=0.07780\left(\frac{R T_{\mathrm{c}}}{P_{\mathrm{c}}}\right)
\end{aligned}
$$

where $a$ and $b$ are the energy and volume parameters. These parameters are determined in conventional way for pure fluids. For the cluster component, the following combining rules are used according to previous investigations for the formation of associated complex through chemical reaction [18-20]:

$$
\left(a_{\mathrm{SF}_{k}} b_{\mathrm{SF}_{k}}\right)^{1 / 2}=k\left(a_{\mathrm{F}} b_{\mathrm{F}}\right)^{1 / 2}+\left(a_{\mathrm{s}} b_{\mathrm{s}}\right)^{1 / 2}
$$

and

$$
b_{\mathrm{SF}_{k}}=k b_{\mathrm{F}}+b_{\mathrm{s}}
$$

The EOS mixture parameters $a_{\mathrm{m}}$ and $b_{\mathrm{m}}$ are evaluated, without using extra empirical parameters, by the simple VDW mixing rules:

$$
\begin{aligned}
& a_{\mathrm{m}}=\sum_{i} \sum_{j} z_{i} z_{j} \sqrt{a_{i} a_{j}} \\
& b_{\mathrm{m}}=\sum_{i} z_{i} b_{j}
\end{aligned}
$$


where the summation is over the supercritical fluid and the solute cluster. The change of Gibbs energy for the cluster formation reaction, $\Delta G^{\mathrm{rxn}}$, is assumed as a simple temperature-dependent form:

$$
\Delta G^{\mathrm{rxn}}\left(T, P_{\mathrm{c}, \mathrm{scf}}\right)=A-B T
$$

Parameters $A$ and $B$ are related to the enthalpy and entropy changes of the cluster formation reaction. In this simplified study, they are taken as empirical parameters for each binary system.

\section{Multi-component system calculations}

Although solid solubility data for single solid in supercritical fluid are abundant in literature, only few experimental results are available for multi-component systems. Lucien and Foster [21] reviewed the solubility data for multi-component systems in supercritical carbon dioxide. Their study recommended that phase behavior of solid mixtures is important but only $<20$ ternary systems are confirmed without partially melted solid mixtures. For multi-component systems with several solids in supercritical fluid, the following cluster formation chemical equilibrium is considered for each solid solute $i$ :

$$
k_{i} \mathrm{~F}+\mathrm{S}_{i} \leftrightarrow \mathrm{S}_{i} \mathrm{~F}_{k_{i}}
$$

where $\mathrm{S}_{i}$ represents solid solute $i, k_{i}$ the average association number of solute $i$ in the supercritical phase, and $\mathrm{S}_{i} \mathrm{~F}_{k_{i}}$ the cluster formed in the supercritical phase. Considering systems with several solid solutes, Eqs. (12) and (13) are written as:

$$
\begin{aligned}
\ln \frac{z_{\mathrm{S}_{i} \mathrm{~F}_{k_{i}}}}{\left(1-k_{i} \sum_{j=1}^{n} z_{\mathrm{S}_{j} \mathrm{~F}_{k_{j}}}\right)}= & k_{i} \ln \frac{\hat{\phi}_{\mathrm{F}}(T, P, z)}{\phi_{\mathrm{F}}\left(T, P_{\mathrm{c}, \mathrm{scf}}\right)}+\frac{V_{\mathrm{s}_{i}}\left(P-P_{\mathrm{c}, \mathrm{scf}}\right)}{R T}-\ln \frac{\hat{\phi}_{\mathrm{S}_{i} \mathrm{~F}_{k_{i}}}(T, P, z)}{\phi_{\mathrm{S}_{i} \mathrm{~F}_{k_{i}}}\left(T, P_{\mathrm{c}, \mathrm{scf}}\right)} \\
& -\frac{\Delta G_{i}^{\mathrm{rxn}}\left(T, P_{\mathrm{c}, \mathrm{scf}}\right)}{R T}+\left(k_{i}-1\right) \ln \frac{P}{P_{\mathrm{c}, \mathrm{scf}}}
\end{aligned}
$$

and

$$
y_{i}=\frac{z_{\mathrm{S}_{i} \mathrm{~F}_{k_{i}}}}{1+\sum_{j=1}^{n} k_{j} z_{\mathrm{S}_{j} \mathrm{~F}_{k j}}}
$$

\section{Extension to fluid mixtures with co-solvent}

With the addition of co-solvent, the association phenomenon becomes more complex. Clusters may be formed as solute-fluid, solute-co-solvent and solute-fluid-co-solvent associates [22]. Experimental data for co-solvent systems are not adequate and thermodynamic correlation is, therefore, very limited. A simplified cluster solvation model is proposed in this study to avoid the complicated equations for various possible cluster forms. It is assumed that effective clusters of solute-fluid are capable of representing all associated fluids. An effective association number as a function of co-solvent concentration, $k_{\text {eff }}$, is used in solubility calculations. One boundary condition for this effective association number is its value for the binary system of the SCF and the pure solid solute. The effective association number is further generalized as a simple function of co-solvent concentration for engineering applications. The working equation for 
the co-solvent system is developed using $\left(1-z_{\mathrm{SF}_{k}}-z_{\mathrm{co}-\text { solvent }}\right)^{k}$ in the denominator of Eq. (10), and applying a Taylor series expansion of the logarithm term at a low solubility condition:

$$
\begin{aligned}
\ln & \frac{z_{\mathrm{SF}_{k}}}{\left(1-z_{\mathrm{co}-\text { solvent }}\right)^{k_{\mathrm{eff}}-1}\left(1-z_{\mathrm{co}-\text { solvent }}-k_{\mathrm{eff}} z_{\mathrm{SF}_{k}}\right)} \\
= & k_{\mathrm{eff}} \ln \frac{\hat{\phi}_{\mathrm{F}}(T, P, z)}{\phi_{\mathrm{F}}\left(T, P_{\mathrm{c}, \mathrm{scf}}\right)}+\frac{V_{\mathrm{s}}\left(P-P_{\mathrm{c}, \mathrm{scf}}\right)}{R T}-\ln \frac{\hat{\phi}_{\mathrm{SF}_{k}}(T, P, z)}{\phi_{\mathrm{SF}_{k}}\left(T, P_{\mathrm{c}, \mathrm{scf}}\right)} \\
& -\frac{\Delta G^{\mathrm{rxn}}\left(T, P_{\mathrm{c}, \mathrm{scf}}\right)}{R T}+\left(k_{\mathrm{eff}}-1\right) \ln \frac{P}{P_{\mathrm{c}, \mathrm{scf}}}
\end{aligned}
$$

and

$$
y=\frac{z_{\mathrm{SF}_{k}}}{z_{\mathrm{F}}+k_{\mathrm{eff}} z_{\mathrm{SF}_{k}}+z_{\mathrm{SF}_{k}}+z_{\mathrm{co}-\text { solvent }}}=\frac{z_{\mathrm{SF}_{k}}}{1+k_{\mathrm{eff}} z_{\mathrm{SF}_{k}}}
$$

Applying Eqs. (27) and (28), solid solubility in a SCF with a co-solvent is calculated.

\section{Results and discussion}

In this study, solid solubilities in supercritical fluid were calculated using the Peng-Robinson EOS with a simplified cluster solvation model. The average association number $k$ and the constants $A$ and $B$ for the Gibbs energy change of cluster formation are taken as adjustable parameters. Total 69 binary systems including highly asymmetric mixtures were investigated. The physical properties of these compounds are taken from literature and listed in Table 1 . The three optimally fitted parameters of $k, A$ and $B$ were determined by minimizing the following objective function of calculated errors for solubility in binary mixtures:

$$
\mathrm{obj}=\frac{100}{n} \sum_{i=1}^{n} \frac{\left|y_{i}^{\mathrm{cal}}-y_{i}^{\mathrm{exp}}\right|}{y_{i}^{\exp }}
$$

The optimally fitted values of three parameters for 69 binary systems are listed in Table 2 . It is observed that an overall absolute average deviation (AAD) for solid solubility calculations is about $8 \%$ using three parameters. Parameter $B$ was further correlated as a function of the other two parameters $k$ and $A$, as well as the critical temperature of the supercritical fluid:

$$
\frac{B}{R k}=-0.0231642\left(\frac{A}{R k T_{\mathrm{c}, \mathrm{scf}}}\right)^{2}+1.76216 \frac{A}{R k T_{\mathrm{c}, \mathrm{scf}}}-11.5288+13.6014 \frac{R k T_{\mathrm{c}, \mathrm{scf}}}{A}
$$

Using Eq. (30), the cluster solvation approach reduces to a model of dual parameters. Fig. 1 shows the calculated solid solubility of triphenylene in supercritical $\mathrm{CO}_{2}$ at various temperatures. With two temperature-independent parameters, the agreement with literature data is satisfactory. Good agreement is also observed for polar solids, as shown in Fig. 2 for the solubility of 2-naphthol in fluoroform.

Comparison of solid solubility calculation results in supercritical fluids from EOS method, semiempirical equations and this simplified cluster solvation model are investigated. Typical results and the overall deviations are shown in Table 3. In the EOS method, the Peng-Robinson equation with VDW mixing rules and one (VDW1) or two (VDW2) parameters was employed. The solid vapor pressures 
Table 1

Physical properties of pure compounds used in this work

\begin{tabular}{|c|c|c|c|c|c|c|c|c|}
\hline Compound & Formula & $T_{\mathrm{c}}(\mathrm{K})$ & $P_{\mathrm{c}}(\mathrm{MPa})$ & $\omega$ & $V_{\mathrm{s}}\left(\mathrm{cm}^{3} \mathrm{~mol}^{-1}\right)$ & $M_{\mathrm{w}}\left(\mathrm{g} \mathrm{mol}^{-1}\right)$ & $\mu(\mathrm{D})$ & $T_{\mathrm{m}}(\mathrm{K})$ \\
\hline Ethane & $\mathrm{C}_{2} \mathrm{H}_{6}$ & $305.4[25]$ & $4.880[25]$ & $0.099[25]$ & - & $30.07[25]$ & $0.0[26]$ & - \\
\hline Ethylene & $\mathrm{C}_{2} \mathrm{H}_{4}$ & 282.4 [25] & $5.040[25]$ & $0.089[25]$ & - & $28.05[25]$ & $0.0[26]$ & - \\
\hline Fluoroform & $\mathrm{CF}_{3} \mathrm{H}$ & $299.3[25]$ & $4.950[25]$ & $0.272[25]$ & - & $70.01[25]$ & $1.62[26]$ & - \\
\hline Monochlorotrifluoromethane & $\mathrm{CClF}_{3}$ & $301.95[25]$ & $3.920[25]$ & $0.180[25]$ & - & $104.46[25]$ & $0.46[26]$ & - \\
\hline Carbon dioxide & $\mathrm{CO}_{2}$ & 304.19 [27] & $7.382[27]$ & 0.228 [27] & - & $44.01[27]$ & $0.18[26]$ & - \\
\hline 1,4-Naphthoquinone & $\mathrm{C}_{10} \mathrm{H}_{6} \mathrm{O}_{2}$ & $877.50[28]$ & $4.067[28]$ & $0.572[28]$ & $111.20[28]$ & 158.16 [29] & $1.20[26]$ & 401.65 [29] \\
\hline 1-Eicosanol & $\mathrm{C}_{20} \mathrm{H}_{42} \mathrm{O}$ & $792.0[27]$ & $1.240[27]$ & 0.937 [27] & $355.21[29]$ & 298.55 [29] & - & $339.25[29]$ \\
\hline 2-Naphthol & $\mathrm{C}_{10} \mathrm{H}_{8} \mathrm{O}$ & $822.4[30]$ & $4.691[30]$ & $0.520[30]$ & $112.70[29]$ & $144.17[29]$ & $1.54[26]$ & 396.15 [29] \\
\hline $2,3,4,5-\mathrm{TCB}$ & $\mathrm{C}_{12} \mathrm{H}_{6} \mathrm{Cl}_{4}$ & $878.0[31]$ & $2.716[31]$ & $0.562[31]$ & $180.0[31]$ & $292.0[31]$ & - & $632.15[29]$ \\
\hline 2,3,4-HCB & $\mathrm{C}_{12} \mathrm{H}_{4} \mathrm{Cl}_{6}$ & $889.0[31]$ & $2.452[31]$ & $0.716[31]$ & $204.0[31]$ & $360.9[31]$ & - & $624.15[29]$ \\
\hline 2,3-Dimethyl naphthalene & $\mathrm{C}_{12} \mathrm{H}_{12}$ & $785.0[4]$ & $3.222[4]$ & $0.424[4]$ & $154.7[4]$ & $156.23[29]$ & $0.69[26]$ & 378.15 [29] \\
\hline 2,6-Dimethyl naphthalene & $\mathrm{C}_{12} \mathrm{H}_{12}$ & $777.0[4]$ & $3.222[4]$ & $0.420[4]$ & $139.2[4]$ & $156.23[29]$ & $0.14[26]$ & 385.15 [29] \\
\hline 2,7-Dimethyl naphthalene & $\mathrm{C}_{12} \mathrm{H}_{12}$ & $771.0[32]$ & $2.910[32]$ & $0.420[32]$ & $136.8[32]$ & $156.23[29]$ & $0.41[26]$ & $370.15[29]$ \\
\hline 3,4-Xylenol & $\mathrm{C}_{8} \mathrm{H}_{10} \mathrm{O}$ & $729.8[25]$ & $4.965[33]$ & $0.576[33]$ & $124.3[33]$ & $122.17[29]$ & $1.77[26]$ & 333.95 [29] \\
\hline 4,4-DCB & $\mathrm{C}_{12} \mathrm{H}_{8} \mathrm{Cl}_{2}$ & $847.5[31]$ & $3.048[31]$ & $0.453[31]$ & $155.0[31]$ & $223.1[31]$ & $2.02[26]$ & 422.45 [29] \\
\hline 5-Methoxyindole & $\mathrm{C}_{9} \mathrm{H}_{9} \mathrm{NO}$ & $745.0[34]$ & $3.550[34]$ & $0.518[34]$ & $127.0[34]$ & $147.18[29]$ & - & 327.15 [29] \\
\hline 6-Caprolactam & $\mathrm{C}_{6} \mathrm{H}_{11} \mathrm{NO}$ & $806.0[27]$ & $4.770[27]$ & $0.477[27]$ & $162.0[29]$ & $113.16[29]$ & $3.88[26]$ & 342.45 [29] \\
\hline 9,10-Anthraqinone & $\mathrm{C}_{14} \mathrm{H}_{8} \mathrm{O}_{2}$ & $900.55[28]$ & $3.372[28]$ & $0.735[28]$ & $145.2[28]$ & 208.23 [29] & $0.0[26]$ & $559.15[29]$ \\
\hline Anthracene & $\mathrm{C}_{14} \mathrm{H}_{10}$ & $869.3[4]$ & $3.486[4]$ & $0.532[4]$ & $142.6[35]$ & $178.23[29]$ & $0.0[26]$ & 488.91 [29] \\
\hline Ascorbic acid & $\mathrm{C}_{6} \mathrm{H}_{8} \mathrm{O}_{6}$ & $790.91[36]$ & $4.419[36]$ & $1.570[36]$ & $106.7[36]$ & $176.13[36]$ & $3.96[26]$ & 465.15 [29] \\
\hline Benzoic acid & $\mathrm{C}_{7} \mathrm{H}_{6} \mathrm{O}_{2}$ & $752.0[25]$ & $4.560[25]$ & $0.620[25]$ & $96.48[29]$ & $122.13[29]$ & $1.80[26]$ & $395.5[29]$ \\
\hline Biphenyl & $\mathrm{C}_{12} \mathrm{H}_{10}$ & $789.0[25]$ & $3.850[25]$ & $0.372[25]$ & $132.0[29]$ & $154.21[29]$ & $0.0[26]$ & 342.08 [29] \\
\hline Chrysene & $\mathrm{C}_{18} \mathrm{H}_{12}$ & $1027.8[37]$ & 2.928 [37] & 0.492 [37] & $179.0[25]$ & $228.30[29]$ & $0.70[26]$ & $492.65[29]$ \\
\hline Dedecyl gallate & $\mathrm{C}_{19} \mathrm{H}_{30} \mathrm{O}_{5}$ & $905.90[36]$ & $1.846[36]$ & $1.20[36]$ & $267.9[36]$ & $338.45[36]$ & - & - \\
\hline Fluorene & $\mathrm{C}_{13} \mathrm{H}_{10}$ & $826.4[38]$ & $2.989[38]$ & $0.406[38]$ & $139.3[35]$ & $116.23[29]$ & $0.83[26]$ & 417.92 [29] \\
\hline Hexamethylbenzene & $\mathrm{C}_{12} \mathrm{H}_{18}$ & $758.0[38]$ & $2.442[38]$ & $0.515[38]$ & $152.7[35]$ & 228.38 [29] & $0.52[26]$ & $438.65[29]$ \\
\hline$o$-HBA & $\mathrm{C}_{7} \mathrm{H}_{6} \mathrm{O}_{3}$ & $739.0[39]$ & $5.180[39]$ & $0.832[39]$ & $95.72[39]$ & $138.12[41]$ & $2.65[26]$ & $432.15[29]$ \\
\hline Indole & $\mathrm{C}_{8} \mathrm{H}_{7} \mathrm{~N}$ & $790.0[34]$ & $4.30[34]$ & 0.374 [34] & 120. [34] & $117.15[29]$ & $2.38[26]$ & $325.65[29]$ \\
\hline Myristic acid & $\mathrm{C}_{14} \mathrm{H}_{28} \mathrm{O}_{2}$ & $756.0[27]$ & $1.702[27]$ & $1.025[27]$ & $257.5[40]$ & 228.38 [29] & $0.76[26]$ & 327.05 [29] \\
\hline Naphthalene & $\mathrm{C}_{10} \mathrm{H}_{8}$ & $748.4[27]$ & $4.063[27]$ & $0.302[27]$ & $110.0[41]$ & $128.17[29]$ & $0.0[26]$ & 353.41 [29] \\
\hline Naproxen & $\mathrm{C}_{14} \mathrm{H}_{14} \mathrm{O}_{3}$ & $807.0[45]$ & $2.420[45]$ & $0.904[45]$ & $178.3[45]$ & $230.3[45]$ & $2.18[26]$ & - \\
\hline Phenanthrene & $\mathrm{C}_{14} \mathrm{H}_{10}$ & $890.0[4]$ & $3.293[4]$ & $0.429[4]$ & $153.0[27]$ & $178.23[29]$ & $0.0[26]$ & 372.39 [29] \\
\hline Phenol & $\mathrm{C}_{6} \mathrm{H}_{6} \mathrm{O}$ & $692.2[25]$ & $6.130[25]$ & $0.450[25]$ & $89.0[38]$ & $94.1[29]$ & $1.46[26]$ & 314.05 [29] \\
\hline$p$-HBA & $\mathrm{C}_{7} \mathrm{H}_{6} \mathrm{O}_{3}$ & 739.0 [39] & $5.180[39]$ & $0.832[39]$ & $95.72[39]$ & $138.12[29]$ & $2.76[26]$ & 487.65 [29] \\
\hline$p$-Quinone & $\mathrm{C}_{6} \mathrm{H}_{4} \mathrm{O}_{2}$ & $747.3[28]$ & $5.002[28]$ & $0.424[28]$ & $82.02[28]$ & $108.10[29]$ & $0.65[26]$ & 388.15 [29] \\
\hline Palmitic acid & $\mathrm{C}_{16} \mathrm{H}_{32} \mathrm{O}_{2}$ & $776.0[27]$ & $1.510[27]$ & $1.083[27]$ & $285.7[40]$ & $256.43[29]$ & $1.76[26]$ & 336.25 [29] \\
\hline Pyrene & $\mathrm{C}_{16} \mathrm{H}_{10}$ & $936.0[25]$ & $2.604[25]$ & $0.509[25]$ & $158.5[35]$ & 202.26 [29] & $0.0[26]$ & $423.77[29]$ \\
\hline Phenylacetic acid & $\mathrm{C}_{8} \mathrm{H}_{8} \mathrm{O}_{2}$ & $775.15[42]$ & $3.860[42]$ & $0.569[42]$ & $110.9[42]$ & 136.14 [29] & $1.77[26]$ & 349.85 [29] \\
\hline Propyl gallate & $\mathrm{C}_{10} \mathrm{H}_{12} \mathrm{O}_{5}$ & $862.87[36]$ & $4.772[36]$ & $0.860[36]$ & $155.0[36]$ & $212.2[36]$ & - & 403.15 [29] \\
\hline Skatole & $\mathrm{C}_{9} \mathrm{H}_{9} \mathrm{~N}$ & $789.0[34]$ & $3.720[34]$ & $0.431[34]$ & $134.0[34]$ & 131.2 [29] & $2.10[26]$ & $370.65[29]$ \\
\hline Stearic acid & $\mathrm{C}_{18} \mathrm{H}_{36} \mathrm{O}_{2}$ & $799.0[25]$ & $1.36[25]$ & $1.084[25]$ & $302.4[37]$ & 284.5 [29] & $1.76[26]$ & $342.45[29]$ \\
\hline Triphenylene & $\mathrm{C}_{18} \mathrm{H}_{12}$ & $1013.6[37]$ & 2.928 [37] & $0.492[37]$ & $175.0[37]$ & 228.29 [29] & $0.0[26]$ & $470.95[29]$ \\
\hline Vanillan & $\mathrm{C}_{8} \mathrm{H}_{8} \mathrm{O}_{3}$ & $777.0[42]$ & $4.010[42]$ & $0.757[42]$ & $144.1[42]$ & 152.16 [29] & $2.45[26]$ & 354.65 [29] \\
\hline 2-Aminobenzoic acid & $\mathrm{C}_{7} \mathrm{H}_{7} \mathrm{NO}_{2}$ & $851.07^{\mathrm{a}}$ & $4.904^{\mathrm{a}}$ & $0.8306^{\mathrm{b}}$ & $97.12[29]$ & $137.14[29]$ & $1.52[26]$ & 419.65 [29] \\
\hline$m$-HBA & $\mathrm{C}_{7} \mathrm{H}_{6} \mathrm{O}_{3}$ & $739.0[39]$ & $5.180[39]$ & $0.832[39]$ & $95.72[39]$ & 138.12 [29] & $2.39[26]$ & $474.65[29]$ \\
\hline DDT & $\mathrm{C}_{14} \mathrm{H}_{9} \mathrm{Cl}_{5}$ & $1097.0[43]$ & $2.290[43]$ & 0.354 [43] & $221.69[47]$ & 354.5 [29] & $1.19[26]$ & 381.65 [29] \\
\hline 2,4-D & $\mathrm{C}_{8} \mathrm{H}_{6} \mathrm{Cl}_{2} \mathrm{O}_{3}$ & $828.0[43]$ & $3.510[43]$ & $1.643[43]$ & $131.27[47]$ & 221.04 [29] & $3.30[26]$ & 411.15 [29] \\
\hline PCP & $\mathrm{C}_{6} \mathrm{Cl}_{5} \mathrm{OH}$ & $816.0[44]$ & $4.11[44]$ & $1.852^{\mathrm{b}}$ & $134.6[44]$ & $266.34[29]$ & $2.14[26]$ & 447.15 [29] \\
\hline НCB & $\mathrm{C}_{6} \mathrm{Cl}_{6}$ & $825.0[27]$ & $2.850[27]$ & $0.5178[27]$ & $181.5[44]$ & $284.78[29]$ & $0.74[26]$ & 501.98 [29] \\
\hline Penicillin G & $\mathrm{C}_{16} \mathrm{H}_{18} \mathrm{O}_{4} \mathrm{~N}_{2} \mathrm{~S}$ & $902.78[46]$ & $2.355[46]$ & $1.3249[46]$ & $226.0[46]$ & $334.0[29]$ & - & - \\
\hline
\end{tabular}

HBA, hydroxybenzoic acid; 2,4-D, 2,4-dichlorophenoxy acetic acid; DDT, 1,1-bis(4-chlorophenyl)-2,2,2-trichloroethane; HCB, hexachlorobenzene; PCP, pentachlorophenol; 4,4-DCB, 4,4-dichlorobiphenyl; 2,3,4-HCB, 2,2,3,3,4,4-hexachlorobiphenyl; 2,3,4,5-TCB, 2,3,4,5-tetrachlorobiphenyl.

${ }^{\text {a }}$ Estimated by Ambrose method [25].

${ }^{\mathrm{b}}$ Estimate by Lee and Kesler method [25]. 
Table 2

Calculated results of solid solubility in supercritical fluid (SCF) using the simplified cluster solvation model

\begin{tabular}{|c|c|c|c|c|c|c|c|c|c|}
\hline SCF & Solid & $T(\mathrm{~K})$ & $P(\mathrm{MPa})$ & Data points & $k$ & $A\left(\mathrm{~J} \mathrm{~mol}^{-1}\right)$ & $B\left(\mathrm{~J} \mathrm{~mol}^{-1} \mathrm{~K}^{-1}\right)$ & AA Dy $(\%)$ & Refs. \\
\hline $\mathrm{CO}_{2}$ & 1,4-Naphthoquinone & $318-343$ & $10-37$ & 18 & 2.749 & 11429.63 & -9.60 & 8.37 & [41] \\
\hline Ethane & 1,4-Naphthoquinone & $318-343$ & $6-37$ & 21 & 3.266 & 14404.75 & -19.21 & 11.42 & [41] \\
\hline Monochlorotrifluormethane & 1,4-Naphthoquinone & $318-328$ & $5-37$ & 12 & 3.494 & 15635.87 & -24.13 & 4.32 & [41] \\
\hline $\mathrm{CO}_{2}$ & 1-Eicosanol & 308.15 & $8-24$ & 7 & 0.306 & 35387.78 & 53.21 & 9.11 & [48] \\
\hline $\mathrm{CO}_{2}$ & 2-Naphthol & $318-343$ & $10-37$ & 19 & 2.684 & 12717.69 & -23.40 & 8.99 & [41] \\
\hline Ethane & 2-Naphthol & $308-343$ & $6-37$ & 27 & 1.672 & 35887.75 & 46.17 & 6.02 & [41] \\
\hline Fluoroform & 2-Naphthol & $328-343$ & $7-37$ & 10 & 3.036 & 14449.53 & -28.58 & 7.68 & [41] \\
\hline Monochlorotrifluormethane & 2-Naphthol & $328-343$ & $7-37$ & 10 & 3.394 & 16650.74 & -34.24 & 3.58 & [41] \\
\hline $\mathrm{CO}_{2}$ & $2,3,4,5-\mathrm{TCB}$ & $308-323$ & $10-46$ & 24 & 2.548 & 12078.84 & -22.25 & 16.14 & [31] \\
\hline $\mathrm{CO}_{2}$ & $2,2,3,3,4,4-\mathrm{HCB}$ & $313-323$ & $15-49$ & 10 & 1.572 & 21271.62 & -2.89 & 18.22 & [31] \\
\hline $\mathrm{CO}_{2}$ & 2,3-Dimethyl naphthalene & $308-328$ & $9-28$ & 15 & 1.804 & 8459.90 & -15.09 & 6.42 & [49] \\
\hline $\mathrm{CO}_{2}$ & 2,6-Dimethyl naphthalene & $308-328$ & $9-28$ & 15 & 1.970 & 9059.60 & -15.09 & 11.18 & [49] \\
\hline $\mathrm{CO}_{2}$ & 2,7-Dimethyl naphthalene & $308-328$ & $9-25$ & 10 & 2.241 & 9598.36 & -10.79 & 4.17 & [32] \\
\hline $\mathrm{CO}_{2}$ & $3,4-X y l e n o l$ & 308.15 & $8-26$ & 7 & 2.602 & 10690.87 & -7.71 & 1.54 & [33] \\
\hline $\mathrm{CO}_{2}$ & 4,4-DCB & $313-323$ & $9-38$ & 17 & 1.512 & 38323.89 & 64.86 & 7.78 & [31] \\
\hline $\mathrm{CO}_{2}$ & 5-Methoxyindole & 308.15 & $7-19$ & 6 & 2.058 & 9999.16 & -19.66 & 2.99 & [34] \\
\hline Ethane & 5-Methoxyindole & 308.15 & $5-20$ & 8 & 1.527 & 10608.41 & -24.24 & 2.98 & [34] \\
\hline Ethylene & 5-Methoxyindole & 308.15 & $7-19$ & 7 & 1.332 & 9072.91 & -21.30 & 3.66 & [34] \\
\hline Fluoroform & 5-Methoxyindole & 308.15 & $5-20$ & 7 & 1.683 & 8475.60 & -18.72 & 4.21 & [34] \\
\hline Ethane & Anthracene & $323-343$ & $10-44$ & 17 & 1.563 & 25761.98 & 13.35 & 5.25 & [50] \\
\hline Ethylene & Anthracene & $323-358$ & $10-48$ & 29 & 1.706 & 22465.78 & 0.74 & 8.47 & [51] \\
\hline Fluoroform & Anthracene & $328-343$ & $9-30$ & 6 & 2.837 & 16243.68 & -39.74 & 12.57 & [41] \\
\hline $\mathrm{CO}_{2}$ & Anthracene & $308-333$ & $10-47$ & 30 & 2.467 & 14042.03 & -33.57 & 14.75 & {$[50,52]$} \\
\hline $\mathrm{CO}_{2}$ & Ascorbic acid & 313.1 & $13-20$ & 4 & 4.199 & 18908.08 & -28.82 & 1.64 & {$[36]$} \\
\hline Ethane & Benzoic acid & $308-328$ & $6-36$ & 23 & 1.176 & 25362.42 & 32.93 & 9.28 & [41] \\
\hline Fluoroform & Benzoic acid & $318-328$ & $6-35$ & 10 & 1.844 & 10317.82 & -25.07 & 11.99 & [41] \\
\hline $\mathrm{CO}_{2}$ & benzoic Acid & $308-328$ & $10-37$ & 33 & 1.075 & 26707.70 & 44.05 & 8.97 & [41] \\
\hline $\mathrm{CO}_{2}$ & Biphenyl & $308-328$ & $10-44$ & 34 & 0.895 & 33768.21 & 78.24 & 11.56 & [53] \\
\hline Ethane & Biphenyl & $308-318$ & $7-28$ & 10 & 0.595 & 26441.52 & 64.28 & 7.86 & [41] \\
\hline $\mathrm{CO}_{2}$ & Chrysene & 308.15 & $8-26$ & 11 & 2.801 & 16869.18 & -40.80 & 3.80 & [54] \\
\hline $\mathrm{CO}_{2}$ & Fluorene & $308-328$ & $7-25$ & 127 & 2.295 & 10466.12 & -16.86 & 7.79 & [55] \\
\hline $\mathrm{CO}_{2}$ & Dedecyl gallate & $313-333$ & $15-25$ & 8 & 4.061 & 19288.74 & -35.74 & 4.38 & [36] \\
\hline Ethylene & Fluorene & $298-343$ & $7-49$ & 21 & 0.998 & 19675.99 & 27.00 & 13.06 & {$[50]$} \\
\hline $\mathrm{CO}_{2}$ & $o$-HBA & $308-328$ & $9-20$ & 26 & 2.365 & 12105.31 & -26.28 & 7.10 & {$[39,56]$} \\
\hline $\mathrm{CO}_{2}$ & Hexamethylbenzene & $323-343$ & $8-49$ & 18 & 1.229 & 30252.95 & 49.29 & 13.06 & {$[50]$} \\
\hline Ethylene & Hexamethylbenzene & 298-343 & $6-48$ & 21 & 1.044 & 21411.80 & 31.76 & 10.54 & [50] \\
\hline Fluoroform & Indole & 308.15 & $5-18$ & 9 & 3.123 & 12341.07 & -5.85 & 3.42 & [34] \\
\hline $\mathrm{CO}_{2}$ & Myristic acid & $308-313$ & $5-37$ & 13 & 1.116 & 51575.57 & 126.95 & 18.58 & {$[40,57]$} \\
\hline Ethane & Naphthalene & $308-318$ & $5-37$ & 26 & 0.631 & 30215.72 & 74.42 & 8.26 & [41] \\
\hline Monochlorotrifluromethane & Naphthalene & $308-328$ & $5-37$ & 21 & 1.976 & 8936.95 & -14.44 & 4.79 & [41] \\
\hline $\mathrm{CO}_{2}$ & Naphthalene & $308-328$ & $8-28$ & 31 & 0.861 & 33857.40 & 79.79 & 7.25 & {$[53,58]$} \\
\hline Fluoroform & Naphthalene & $308-328$ & $6-35$ & 18 & 2.264 & 9568.90 & -11.23 & 4.59 & [41] \\
\hline $\mathrm{CO}_{2}$ & Naproxen & $313-333$ & $8-20$ & 18 & 3.101 & 16323.38 & -36.78 & 7.38 & [45] \\
\hline $\mathrm{CO}_{2}$ & $p$-HBA & $318-328$ & $10-21$ & 12 & 2.460 & 25531.10 & -28.08 & 7.43 & [39] \\
\hline $\mathrm{CO}_{2}$ & $p$-Quinone & $308-318$ & $8-30$ & 18 & 0.745 & 28326.49 & 65.85 & 14.39 & [28] \\
\hline $\mathrm{CO}_{2}$ & Palmitic acid & 313.15 & $8-25$ & 9 & 0.545 & 46794.90 & 99.74 & 10.98 & [40] \\
\hline $\mathrm{CO}_{2}$ & Penicillin G & $313-333$ & $15-35$ & 15 & 4.129 & 19104.74 & -32.55 & 12.92 & [46] \\
\hline $\mathrm{CO}_{2}$ & Phenanthrene & $308-328$ & $7-26$ & 127 & 2.957 & 12696.38 & -14.58 & 7.79 & {$[55]$} \\
\hline Ethane & Phenanthrene & $313-328$ & $6-41$ & 23 & 0.952 & 21630.27 & 30.92 & 10.49 & {$[41,50]$} \\
\hline Ethylene & Phenanthrene & $298-343$ & $5-28$ & 30 & 1.134 & 24029.36 & 37.65 & 8.48 & {$[51]$} \\
\hline Fluoroform & Phenanthrene & $318-328$ & $8-37$ & 8 & 2.736 & 12667.80 & -23.22 & 4.26 & [41] \\
\hline $\mathrm{CO}_{2}$ & Phenylacetic acid & $308-318$ & $8-19$ & 24 & 0.847 & 40776.40 & 100.96 & 7.11 & [42] \\
\hline Monochlorotrifluromethane & Phenanthrene & $318-328$ & $7-31$ & 8 & 2.307 & 11908.33 & -26.63 & 3.33 & [41] \\
\hline $\mathrm{CO}_{2}$ & Phenol & $309-333$ & $7-24$ & 41 & 0.775 & 26415.37 & 51.73 & 7.83 & [59] \\
\hline $\mathrm{CO}_{2}$ & Pyrene & $308-328$ & $8-25$ & 118 & 2.904 & 13784.48 & -25.50 & 8.98 & [55] \\
\hline Ethylene & Pyrene & $318-343$ & $10-49$ & 14 & 1.161 & 18207.11 & 12.01 & 13.57 & [50] \\
\hline $\mathrm{CO}_{2}$ & Propyl gallate & 313-333 & $15-25$ & 8 & 3.620 & 18912.70 & -42.29 & 3.84 & [36] \\
\hline $\mathrm{CO}_{2}$ & Skatole & 308.15 & $7-19$ & 8 & 2.840 & 11808.48 & -9.95 & 9.00 & [34] \\
\hline
\end{tabular}


Table 2 (Continued)

\begin{tabular}{|c|c|c|c|c|c|c|c|c|c|}
\hline SCF & Solid & $T(\mathrm{~K})$ & $P(\mathrm{MPa})$ & Data points & $k$ & $A\left(\mathrm{~J} \mathrm{~mol}^{-1}\right)$ & $B\left(\mathrm{~J} \mathrm{~mol}^{-1} \mathrm{~K}^{-1}\right)$ & AA Dy $(\%)$ & Refs. \\
\hline Ethane & Skatole & 308.15 & $4-19$ & 8 & 2.317 & 10608.72 & -17.02 & 5.83 & [34] \\
\hline Ethylene & Skatole & 308.15 & $7-20$ & 8 & 2.047 & 9150.60 & -18.95 & 4.91 & [34] \\
\hline Fluoroform & Skatole & 308.15 & $5-19$ & 8 & 2.868 & 11820.55 & -11.03 & 2.86 & {$[34]$} \\
\hline $\mathrm{CO}_{2}$ & Stearic acid & 308.15 & $9-24$ & 8 & 1.069 & 71462.05 & 180.05 & 7.92 & [48] \\
\hline $\mathrm{CO}_{2}$ & Triphenylene & $308-328$ & $8-26$ & 28 & 2.953 & 15778.21 & -36.12 & 4.42 & {$[54]$} \\
\hline $\mathrm{CO}_{2}$ & Vanillan & $308-318$ & $8-20$ & 33 & 2.623 & 11304.44 & -13.31 & 4.86 & [42] \\
\hline $\mathrm{CO}_{2}$ & 9,10 -Anthraqinone & $308-318$ & $8-30$ & 17 & 1.716 & 28697.84 & 16.67 & 9.93 & [28] \\
\hline $\mathrm{CO}_{2}$ & 2-Aminobenzoic acid & 308 & $12-35$ & 6 & 4.085 & 17575.33 & -20.49 & 2.83 & {$[60]$} \\
\hline $\mathrm{CO}_{2}$ & $m$-HBA & $318-328$ & $10-21$ & 12 & 2.404 & 25090.52 & -27.10 & 7.58 & {$[61]$} \\
\hline $\mathrm{CO}_{2}$ & DDT & $313-333$ & $10-22$ & 18 & 3.036 & 13297.51 & -17.48 & 7.10 & [43] \\
\hline $\mathrm{CO}_{2}$ & $2,4-\mathrm{D}$ & $313-333$ & $10-22$ & 16 & 4.311 & 18753.66 & -23.61 & 3.55 & {$[43]$} \\
\hline Grand average & & & & 1439 & & & & 8.29 & \\
\hline
\end{tabular}

were taken from literature. Those unavailable sublimation pressures were also taken as optimally fitted parameters in the following form:

$$
\ln P^{\mathrm{sat}}(\mathrm{Pa})=C_{1}-\frac{C_{2}}{T(\mathrm{~K})}
$$

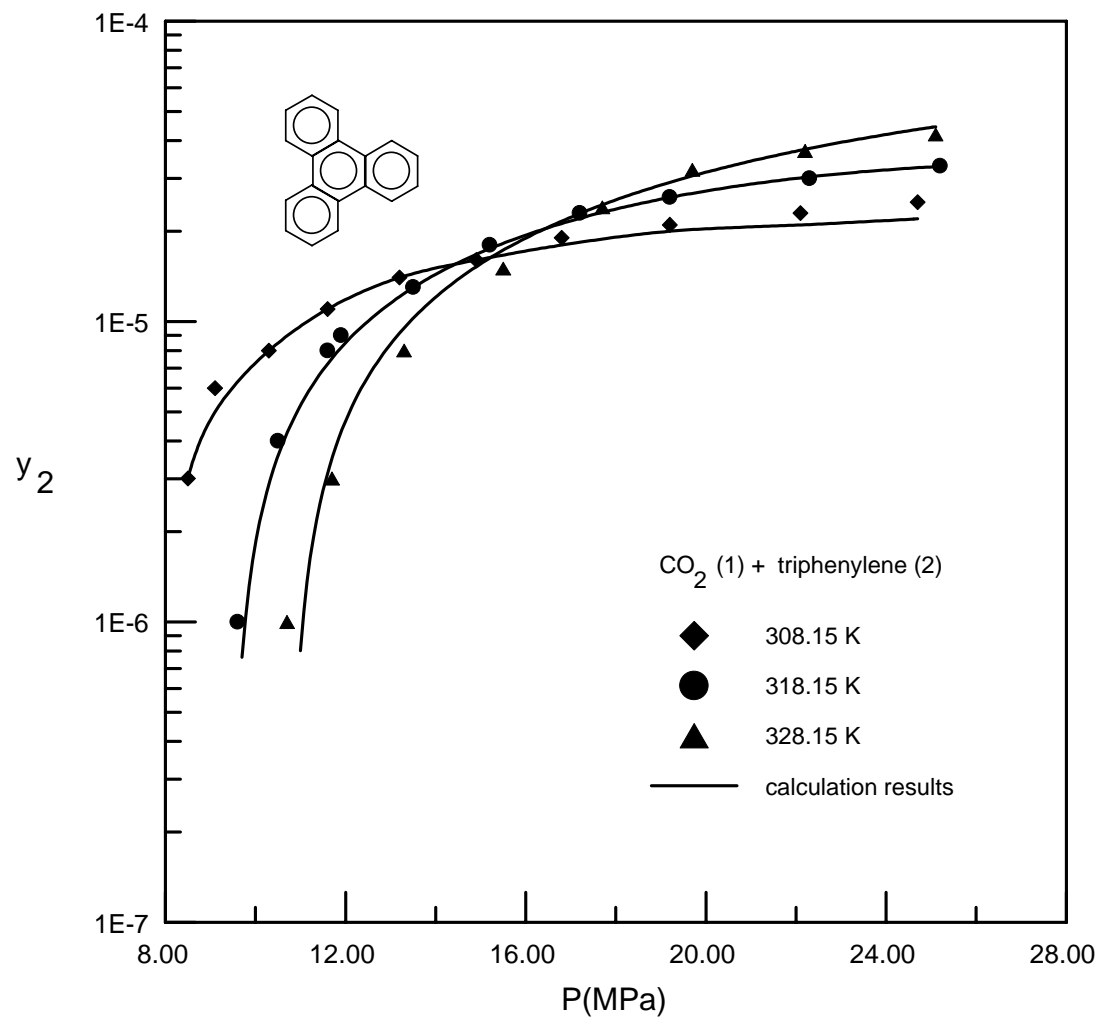

Fig. 1. Calculation results for the solid solubility of triphenylene in supercritical carbon dioxide with two parameters of the simplified cluster solvation model. 


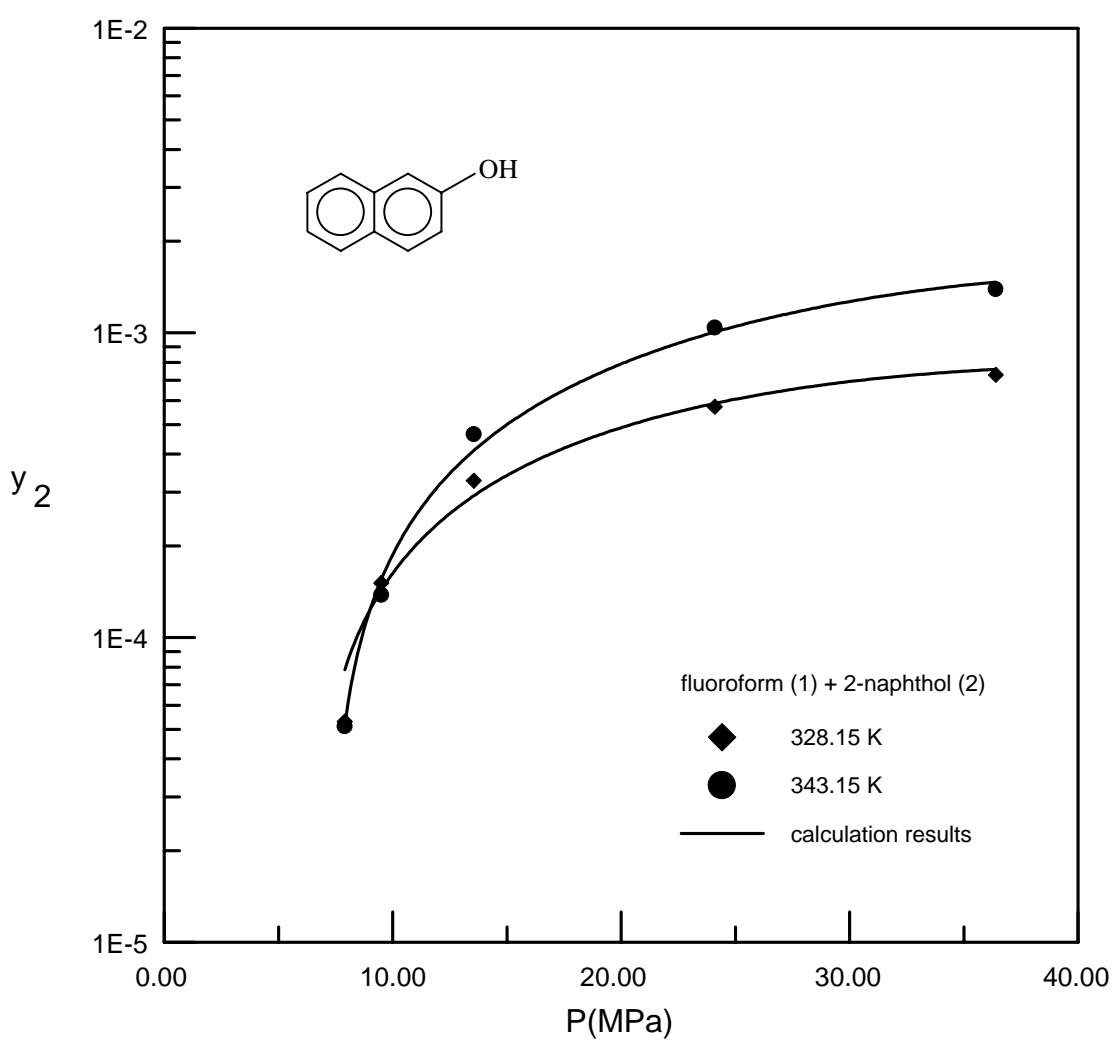

Fig. 2. Calculation results for the solid solubilities of 2-naphthol in supercritical fluoroform with two parameters of the simplified cluster solvation model.

where $C_{1}$ and $C_{2}$ are two empirically adjusted parameters. In the semi-empirical equation approach, the Chrastil [23] and Mendez-Santiago and Teja [8] equations are employed in this comparison.

It is shown in Table 3 that an AAD about $20.77 \%$ is observed using the Peng-Robinson EOS with VDW1 mixing rules. With VDW2 mixing rules, the Peng-Robinson equation method yields an AAD of $12.35 \%$. Mendez-Santiago and Teja equation with three parameters gives an AAD of $14.03 \%$, and an $\mathrm{AAD}$ of $10.21 \%$ is obtained from the Chrastil equation also with three parameters. The simplified cluster solvation model with three parameters $(k, A$ and $B)$ gives an AAD about 8.29\%. When one parameter $(B)$ is generalized, the cluster solvation model shows an AAD of $9.87 \%$. The grand average deviation is smaller using this simplified cluster solvation model. The number of adjustable parameters is reduced to two without substantial change in accuracy. Since the calculated deviation is around $10 \%$, it is satisfactory with respect to experimental accuracy. It is recognized that several good correlative equations can provide comparative results for binary systems. Most of them, however, cannot be directly employed for multi-component or co-solvent systems.

This simplified cluster solvation model is further applied to predict the solid solubilities of multicomponent systems. The values of $k, A$, and $B$ are kept as the same from the binary system calculations. The prediction results are presented in Table 4 for 19 ternary systems with two solid solutes and confirmed solid-vapor equilibrium [21]. The overall AAD for the prediction is about $20.68 \%$ with a peak deviation 
Table 3

Comparison of the calculated solid solubility in supercritical fluid using different methods

\begin{tabular}{|c|c|c|c|c|c|c|c|c|c|}
\hline \multirow[t]{2}{*}{ SCF } & \multirow[t]{2}{*}{ Solid } & \multirow[t]{2}{*}{ Data points } & \multicolumn{6}{|c|}{ AA Dy $(\%)$} & \multirow[t]{2}{*}{ Ref. } \\
\hline & & & A & B & $\mathrm{C}$ & $\mathrm{D}$ & $\mathrm{E}$ & $\mathrm{F}$ & \\
\hline Monochlorotrifluormethane & 1,4-Naphthoquinone & 12 & 22.95 & 13.23 & 10.62 & 10.39 & 4.32 & 5.38 & [41] \\
\hline Fluoroform & 2-Naphthol & 10 & 36.83 & 10.42 & 11.55 & 19.51 & 7.68 & 7.68 & [41] \\
\hline $\mathrm{CO}_{2}$ & 3,4-Xylenol & 7 & 25.53 & 5.92 & 0.89 & 4.10 & 1.54 & 1.54 & [33] \\
\hline $\mathrm{CO}_{2}$ & 5-Methoxyindole & 6 & 7.71 & 4.33 & 6.98 & 4.93 & 2.99 & 2.99 & [34] \\
\hline Ethylene & 5-Methoxyindole & 7 & 16.99 & 2.91 & 3.54 & 2.64 & 3.66 & 3.66 & [34] \\
\hline $\mathrm{CO}_{2}$ & Ascorbic acid & 4 & 11.09 & 2.42 & 2.02 & 1.72 & 1.64 & 1.64 & [36] \\
\hline $\mathrm{CO}_{2}$ & Benzoic acid & 33 & 25.45 & 14.26 & 10.88 & 14.27 & 8.97 & 9.71 & [41] \\
\hline Fluoroform & Indole & 12 & 40.53 & 17.56 & 5.73 & 7.94 & 3.42 & 3.42 & [34] \\
\hline Fluoroform & Naphthalene & 18 & 27.47 & 11.76 & 14.61 & 16.17 & 4.59 & 5.26 & [41] \\
\hline $\mathrm{CO}_{2}$ & Penicillin G & 15 & 33.80 & 11.39 & 32.40 & 12.94 & 12.92 & 12.92 & [46] \\
\hline $\mathrm{CO}_{2}$ & Phenanthrene & 127 & 22.56 & 14.32 & 10.65 & 18.06 & 7.79 & 8.77 & {$[55]$} \\
\hline $\mathrm{CO}_{2}$ & Phenylacetic acid & 24 & 31.18 & 19.73 & 9.72 & 12.93 & 7.11 & 11.50 & [42] \\
\hline $\mathrm{CO}_{2}$ & Propyl gallate & 8 & 13.07 & 5.16 & 2.05 & 2.05 & 3.84 & 6.98 & [36] \\
\hline Ethane & Skatole & 8 & 21.48 & 8.10 & 11.25 & 10.12 & 5.83 & 5.82 & [34] \\
\hline $\mathrm{CO}_{2}$ & DDT & 18 & 11.20 & 11.01 & 8.81 & 21.01 & 7.10 & 9.05 & [43] \\
\hline $\begin{array}{c}\text { Grand average over } \\
1439 \text { data points }\end{array}$ & & & 20.77 & 12.35 & 10.12 & 14.03 & 8.29 & 9.87 & \\
\hline
\end{tabular}

A, PR EOS with VDW1 mixing rules; B, PR EOS with VDW2 mixing rules; C, Chrastil equation (three parameters); D, Mendez-Santiago and Teja equation (three parameters); E, this work with three parameters; F, this work with two parameters.

Table 4

Prediction results of the solid solubility with two solids in supercritical fluids

\begin{tabular}{|c|c|c|c|c|c|c|c|c|}
\hline SCF & Solid (1) & Solid (2) & $\begin{array}{l}\text { Data } \\
\text { points }\end{array}$ & $T(\mathrm{~K})$ & $P(\mathrm{MPa})$ & $\begin{array}{l}\mathrm{AA} \mathrm{Dy}_{1} \\
(\%)\end{array}$ & $\begin{array}{l}\mathrm{AA} \mathrm{Dy}_{2} \\
(\%)\end{array}$ & Ref. \\
\hline $\mathrm{CO}_{2}$ & 2-Aminobenzoic acid & Anthracene & 5 & 308 & $12-35$ & 9.42 & 10.54 & {$[62]$} \\
\hline $\mathrm{CO}_{2}$ & Anthracene & 2-Naphthol & 6 & 308 & $12-35$ & 15.92 & 20.52 & {$[62]$} \\
\hline $\mathrm{CO}_{2}$ & Anthracene & Phenanthrene & 10 & $308-318$ & $10-25$ & 22.46 & 21.21 & {$[52]$} \\
\hline $\mathrm{CO}_{2}$ & Benzoic acid & Hexamethylbenzene & 6 & 308 & $10-35$ & 23.49 & 10.66 & [62] \\
\hline $\mathrm{CO}_{2}$ & $2,4-\mathrm{D}$ & DDT & 6 & 313 & $10-21$ & 5.80 & 15.33 & [43] \\
\hline $\mathrm{CO}_{2}$ & 2,3-Dimethyl naphthalene & 2,6-Dimethyl naphthalene & 18 & $308-318$ & $11-28$ & 9.07 & 12.25 & {$[63]$} \\
\hline $\mathrm{CO}_{2}$ & 2,6-Dimethyl naphthalene & 2,7-Dimethyl naphthalene & 6 & 308 & $9-25$ & 1.62 & 10.76 & [32] \\
\hline $\mathrm{CO}_{2}$ & 2,3-Dimethyl naphthalene & Naphthalene & 6 & 308 & $12-28$ & 37.75 & 24.88 & [63] \\
\hline $\mathrm{CO}_{2}$ & 2,6-Dimethyl naphthalene & Naphthalene & 9 & $303-313$ & $24-31$ & 21.54 & 28.69 & [64] \\
\hline $\mathrm{CO}_{2}$ & 2,3-Dimethyl naphthalene & Phenanthrene & 10 & $308-318$ & $11-28$ & 25.82 & 18.07 & {$[63]$} \\
\hline $\mathrm{CO}_{2}$ & 2,6-Dimethyl naphthalene & Phenanthrene & 6 & 308 & $11-28$ & 5.52 & 32.11 & {$[63]$} \\
\hline $\mathrm{CO}_{2}$ & $o$-HBA & $m$-HBA & 6 & 318 & $10-21$ & 10.87 & 50.40 & {$[61]$} \\
\hline $\mathrm{CO}_{2}$ & $o$-HBA & $p$-HBA & 12 & $318-328$ & $10-21$ & 5.97 & 54.92 & [39] \\
\hline $\mathrm{CO}_{2}$ & $m$-HBA & $p$-HBA & 12 & $318-328$ & $10-21$ & 7.29 & 11.76 & {$[61]$} \\
\hline $\mathrm{CO}_{2}$ & Naphthalene & Phenanthrene & 14 & 308 & $8-28$ & 11.25 & 43.48 & {$[52]$} \\
\hline $\mathrm{CO}_{2}$ & Naphthalene & Benzoic acid & 10 & $308-318$ & $12-28$ & 17.26 & 48.58 & {$[63]$} \\
\hline $\mathrm{CO}_{2}$ & Phenanthrene & Benzoic acid & 5 & 308.15 & $12-28$ & 43.52 & 19.72 & [63] \\
\hline $\mathrm{CO}_{2}$ & 2-Naphthol & Phenanthrene & 16 & $308-328$ & $13-35$ & 15.72 & 14.78 & [64] \\
\hline Fluoroform & Naphthalene & Phenanthrene & 6 & 308 & $6-27$ & 15.85 & 40.19 & {$[65]$} \\
\hline
\end{tabular}

Total number of data points $=169, \mathrm{AA} \mathrm{Dy}_{1}=15.42 \%, \mathrm{AA} \mathrm{Dy}_{2}=25.94 \%$, overall $\mathrm{AA} \mathrm{Dy}=20.68 \%$. 


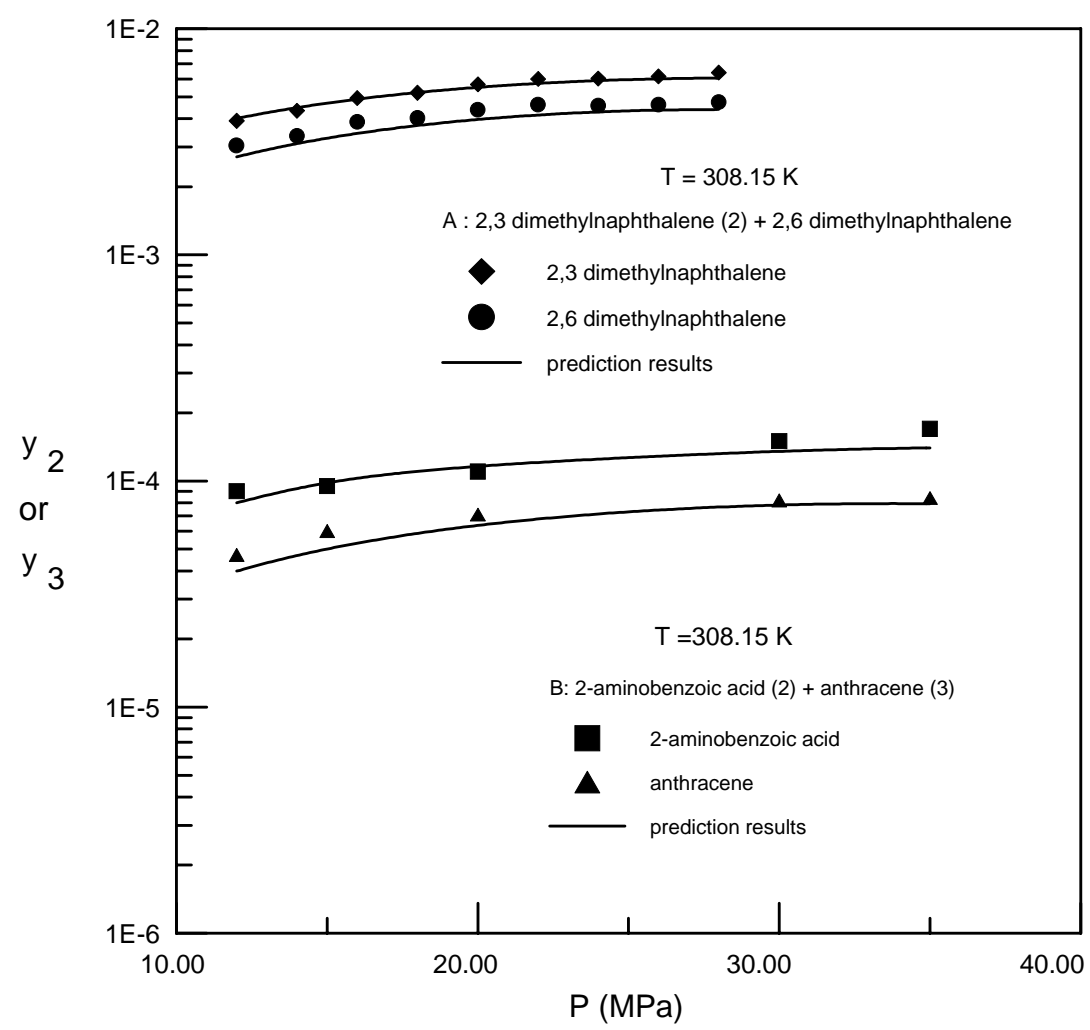

Fig. 3. Prediction results for solid solubility of two ternary mixtures with two solid solutes in supercritical carbon dioxide using the simplified cluster solvation model.

around $50 \%$. About $75 \%$ of the calculated results in this study show an AAD $<25 \%$, which is considered satisfactory for predictions. A graphical presentation of the predictions for two ternary mixtures is shown in Fig. 3. On the other hand, when EOS method is employed in predicting the solid solubility on ternary systems by directly using the binary interaction parameters, a higher AAD of $32 \%$ with peak error up to $96 \%$ is observed. Experimental data for one system with three solid solutes is found in literature, and the predicted solubility using this simplified cluster solvation model is shown in Table 5. Without introducing any additional parameter, this model can still estimate the very low solid solubility (about $10^{-7}$

Table 5

Calculation results of three solids in supercritical carbon dioxide

\begin{tabular}{|c|c|c|c|c|c|c|c|c|}
\hline \multirow[t]{2}{*}{ Solid } & \multirow[t]{2}{*}{ Data points } & \multirow[t]{2}{*}{$T(\mathrm{~K})$} & \multirow[t]{2}{*}{$P(\mathrm{MPa})$} & \multicolumn{2}{|c|}{ Predicted } & \multicolumn{2}{|c|}{ Correlated } & \multirow[t]{2}{*}{ Ref. } \\
\hline & & & & $k$ & AA Dy $(\%)$ & $k$ & AA Dy $(\%)$ & \\
\hline$o-\mathrm{HBA}$ & 6 & 318 & $10-20$ & 2.365 & 9.67 & 2.398 & 3.25 & [61] \\
\hline$m$-HBA & & & & 2.404 & 50.16 & 2.249 & 9.79 & \\
\hline$p$-HBA & & & & 2.460 & 54.09 & 2.290 & 13.62 & \\
\hline
\end{tabular}


mole fraction) on the multi-component system. If the association number for each solid solute is optimally fitted, the correlated result with AAD around 10\% is obtained as shown in Table 5. It is also observed that the best fitted $k$ values only slightly deviate from those of the binary correlation results. It is reasonable to assume that the average association number around a solid changes with the addition of another solid. One major assumption for calculating the solid solubility in supercritical fluid is that the solid components are almost independent of each other, in this way, the change in average association number for multiple solid system is not obvious. Applying the simplified cluster solvation model developed in this study, similar conclusion is observed in Table 5, and it makes the prediction of solid solubility possible using directly the binary parameters. Most of the binary mixture data correlated with this cluster solvation model are either isothermal or in a small temperature range of $20 \mathrm{~K}$. Temperature effect on the correlated parameters and prediction results requires further investigation.

This simplified cluster solvation model is finally employed to calculate the solid solubility in supercritical fluid with co-solvent. The effective association number $k_{\text {eff }}$ for each solid is obtained by regressing the experimental solubility data. Parameters $A$ and $B$ in the Gibbs energy change of the cluster formation reaction also require modification. For each solid solute, we first apply the binary system parameters and divide $A$ and $B$ by $k$ to obtain the contributions from each association site. The resulting values are then multiplied by $k_{\text {eff }}$ to yield the effective $A$ and $B$ parameters for co-solvent systems. The calculation results are presented in Table 6 . The overall AAD is about $7.2 \%$ over 232 data points in this study. Fig. 4

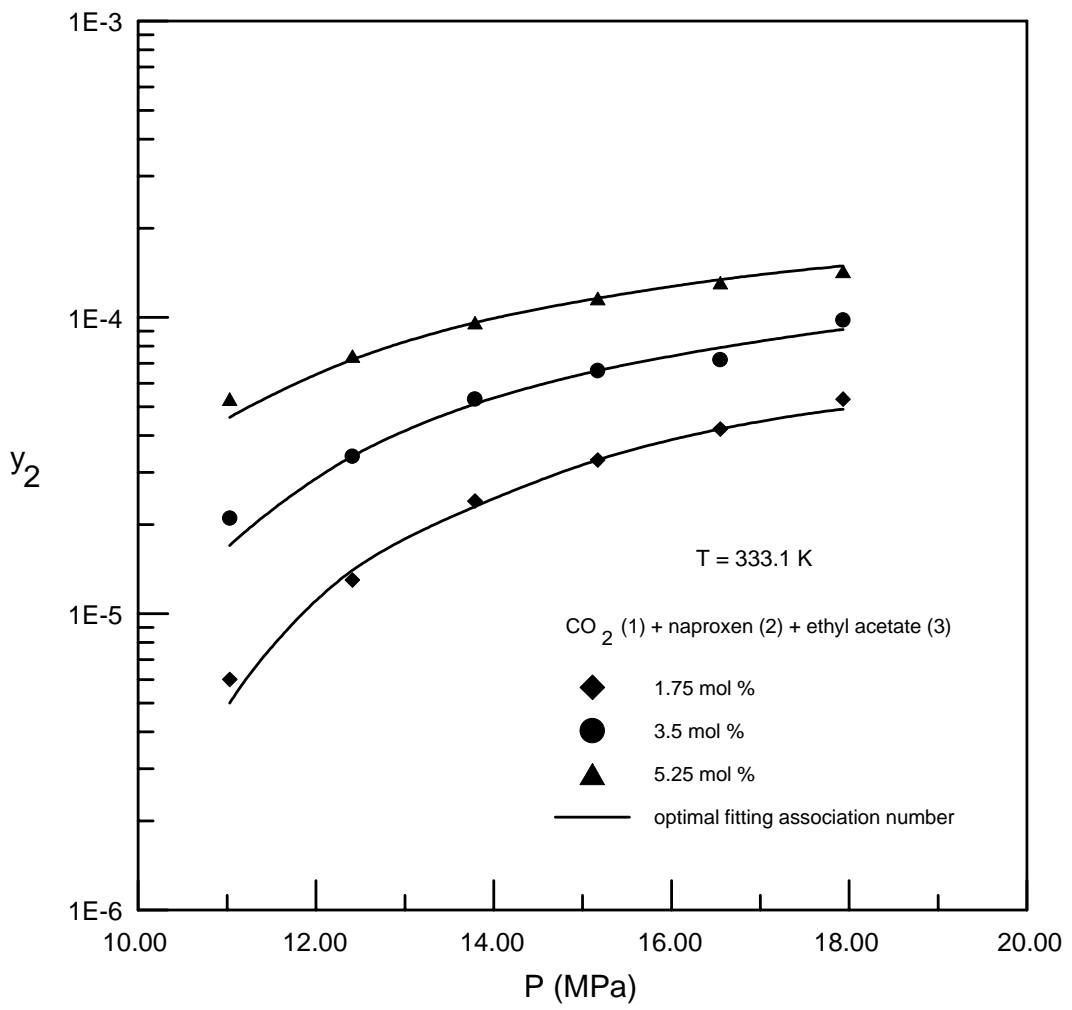

Fig. 4. Correlation results for the solid solubility of naproxen with various concentrations of ethyl acetate co-solvent in supercritical carbon dioxide. 
Table 6

Calculation results of the solid solubility in supercritical fluid with co-solvents

\begin{tabular}{|c|c|c|c|c|c|c|c|c|}
\hline $\mathrm{SCF}$ & Co-solvent (mol\%) & Solid & Data points & $T(\mathrm{~K})$ & $P(\mathrm{MPa})$ & $k_{\mathrm{eff}}$ & $\begin{array}{l}\mathrm{AA} \mathrm{Dy}_{1} \\
(\%)\end{array}$ & Ref. \\
\hline $\mathrm{CO}_{2}$ & Acetone $(3.5 \%)$ & 2-Aminobenzoic acid & 6 & 308 & $9-30$ & 3.580 & 8.45 & [60] \\
\hline $\mathrm{CO}_{2}$ & Octane $(3.5 \%)$ & 2-Aminobenzoic acid & 6 & 308 & $9-30$ & 3.118 & 4.82 & {$[60]$} \\
\hline $\mathrm{CO}_{2}$ & Methanol (3.5\%) & 2-Naphthol & 5 & 308 & $12-35$ & 2.036 & 3.64 & {$[60]$} \\
\hline $\mathrm{CO}_{2}$ & Methanol (7\%) & 2-Naphthol & 6 & 308 & $12-35$ & 1.688 & 7.27 & {$[62]$} \\
\hline $\mathrm{CO}_{2}$ & Methanol (3.5\%) & Anthracene & 5 & 308 & $12-30$ & 2.389 & 8.04 & {$[62]$} \\
\hline $\mathrm{CO}_{2}$ & Acetone $(3.5 \%)$ & Benzoic acid & 7 & 308 & $10-33$ & 0.889 & 8.84 & [62] \\
\hline $\mathrm{CO}_{2}$ & Methanol (3.5\%) & Benzoic acid & 18 & $308-328$ & $9-35$ & 0.752 & 10.85 & {$[62]$} \\
\hline $\mathrm{CO}_{2}$ & Octane $(3.5 \%)$ & Benzoic acid & 5 & 308 & $10-30$ & 0.905 & 5.35 & {$[60]$} \\
\hline $\mathrm{CO}_{2}$ & Octane $(3.5 \%)$ & Hexamethylbenzene & 6 & 308 & $12-35$ & 1.083 & 8.46 & {$[66]$} \\
\hline $\mathrm{CO}_{2}$ & Pentane $(3.5 \%)$ & Hexamethylbenzene & 6 & 308 & $10-35$ & 1.129 & 7.87 & [66] \\
\hline $\mathrm{CO}_{2}$ & Acetone $(1.75 \%)$ & Naproxen & 7 & 333 & $11-20$ & 2.881 & 5.21 & [45] \\
\hline $\mathrm{CO}_{2}$ & Acetone $(3.5 \%)$ & Naproxen & 20 & $318-333$ & $8-18$ & 2.731 & 5.48 & {$[45]$} \\
\hline $\mathrm{CO}_{2}$ & Acetone $(5.25 \%)$ & Naproxen & 6 & 333 & $11-18$ & 2.564 & 2.39 & [45] \\
\hline $\mathrm{CO}_{2}$ & Ethyl acetate $(1.75 \%)$ & Naproxen & 6 & 333 & $11-18$ & 2.972 & 4.71 & [45] \\
\hline $\mathrm{CO}_{2}$ & Ethyl acetate $(3.5 \%)$ & Naproxen & 6 & 333 & $11-18$ & 2.780 & 6.73 & [45] \\
\hline $\mathrm{CO}_{2}$ & Ethyl acetate $(5.25 \%)$ & Naproxen & 6 & 333 & $11-18$ & 2.632 & 3.81 & [45] \\
\hline $\mathrm{CO}_{2}$ & Ethanol $(1.75 \%)$ & Naproxen & 6 & 333 & $11-18$ & 2.715 & 8.68 & [45] \\
\hline $\mathrm{CO}_{2}$ & Ethanol $(3.5 \%)$ & Naproxen & 12 & $323-333$ & $11-18$ & 2.467 & 10.99 & [45] \\
\hline $\mathrm{CO}_{2}$ & Ethanol $(5.25 \%)$ & Naproxen & 6 & 333 & $11-18$ & 2.236 & 1.26 & [45] \\
\hline $\mathrm{CO}_{2}$ & Methanol (1.75\%) & Naproxen & 6 & 333 & $11-18$ & 2.738 & 7.43 & [45] \\
\hline $\mathrm{CO}_{2}$ & Methanol (3.5\%) & Naproxen & 14 & $323-333$ & $11-18$ & 2.414 & 4.19 & [45] \\
\hline $\mathrm{CO}_{2}$ & Methanol (5.25\%) & Naproxen & 6 & 333 & $11-18$ & 2.269 & 2.75 & [45] \\
\hline $\mathrm{CO}_{2}$ & 1-Propanol (1.75\%) & Naproxen & 5 & 333 & $11-18$ & 2.655 & 16.60 & [45] \\
\hline $\mathrm{CO}_{2}$ & 1-Propanol (3.5\%) & Naproxen & 5 & 333 & $11-18$ & 2.320 & 9.95 & [45] \\
\hline $\mathrm{CO}_{2}$ & 1-Propanol (5.25\%) & Naproxen & 5 & 333 & $11-18$ & 2.148 & 4.18 & [45] \\
\hline $\mathrm{CO}_{2}$ & 2-Propanol (1.75\%) & Naproxen & 6 & 333 & $11-18$ & 2.665 & 8.91 & [45] \\
\hline $\mathrm{CO}_{2}$ & 2-Propanol (3.5\%) & Naproxen & 11 & $323-333$ & $11-18$ & 2.412 & 10.69 & [45] \\
\hline $\mathrm{CO}_{2}$ & 2-Propanol (5.25\%) & Naproxen & 5 & 333 & $11-18$ & 2.162 & 3.71 & [45] \\
\hline $\mathrm{CO}_{2}$ & Octane $(3.5 \%)$ & Phenanthrene & 7 & 308 & $12-35$ & 2.575 & 5.99 & [66] \\
\hline $\mathrm{CO}_{2}$ & Octane $(5.25 \%)$ & Phenanthrene & 5 & 308 & $12-35$ & 2.424 & 9.39 & [66] \\
\hline $\mathrm{CO}_{2}$ & Octane $(7 \%)$ & Phenanthrene & 6 & 308 & $12-35$ & 2.295 & 3.84 & [66] \\
\hline $\mathrm{CO}_{2}$ & $n$-Pentane $(3.5 \%)$ & Phenanthrene & 6 & 308 & $12-35$ & 2.751 & 14.78 & [66] \\
\hline Grand average & & & 232 & & & & 7.20 & \\
\hline
\end{tabular}

presents the calculation results of naproxen with various concentrations of the ethyl acetate cosolvent in supercritical carbon dioxide. The calculation results are in satisfactory agreement with experimental data. Fig. 5 shows the concentration dependence of the effective association numbers where a nearly linear relation is observed. Since the boundary point at zero co-solvent concentration is the $k$-value of binary system, the only additional parameter is the slope of each curve shown in Fig. 5. Using the generalized correlation of $k_{\text {eff }}$ (for example, it equals to $k_{\text {eff }}=2.957-0.096 z_{3}$ for the $\mathrm{CO}_{2}(1)+$ phenanthrene (2) + octane (3) ternary system, $z_{3}$ is the mole fraction of the co-solvent octane), the overall calculated error for solid solubility is around $12 \%$, with a peak error around $20 \%$. This result is considered satisfactory, and is comparable to a recent study of Koga et al. [24] with one more parameter. 


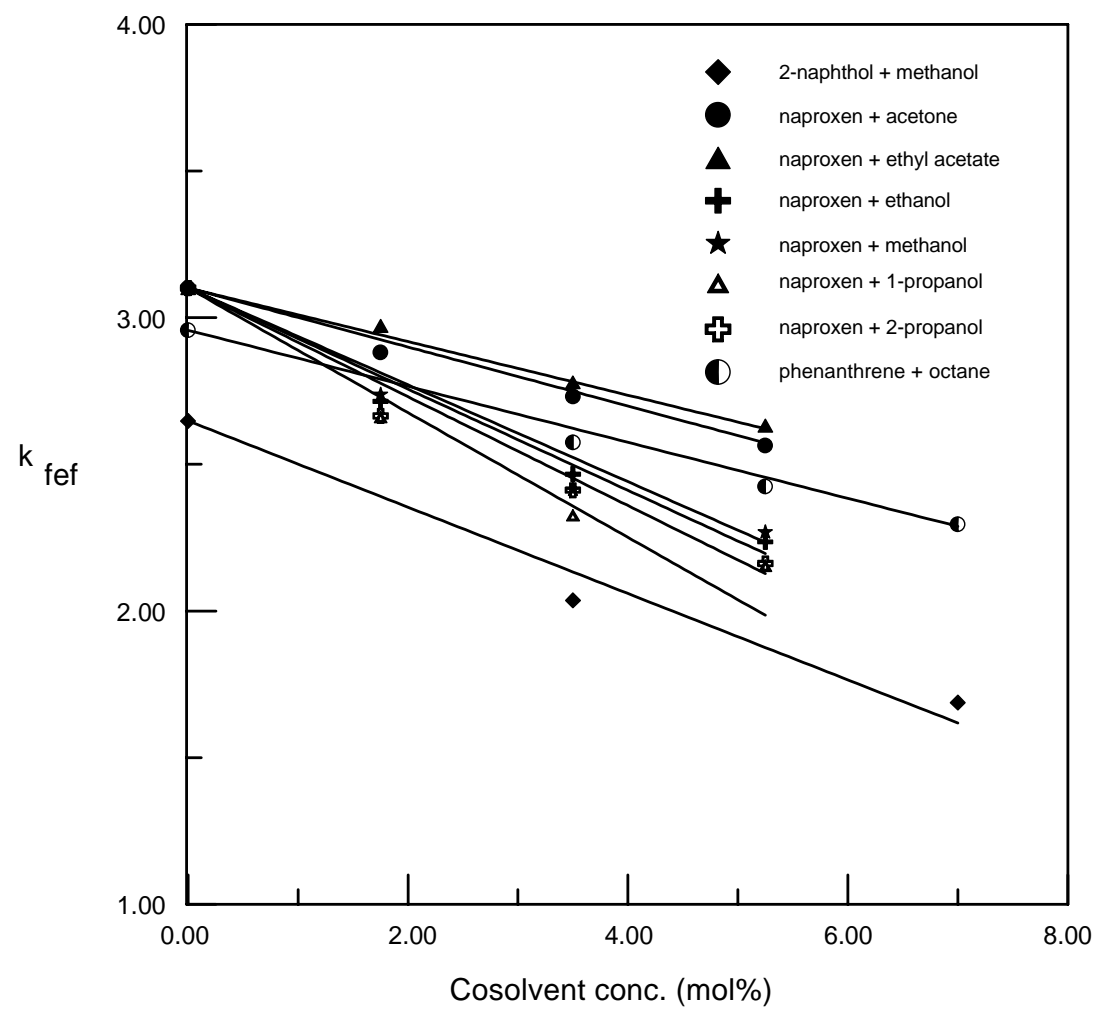

Fig. 5. Plot of the association number against the co-solvent concentration in supercritical carbon dioxide using the simplified cluster solvation model.

The feasibility for multi-component and co-solvent system calculations is the major advantage using this simplified cluster solvation model.

\section{Conclusion}

A simplified cluster solvation model is presented in this study to calculate the solid solubility for binary, multi-component, and co-solvent systems with various supercritical fluids. Two parameters are used for binary systems and their values are directly applicable to ternary- and multiple-component mixtures. Comparison with other methods also indicates that this simplified cluster solvation model yields better accuracy with less adjustable parameters. The co-solvent systems have been investigated using a simplified approach with an effective association number. The calculation results for co-solvent systems are again satisfactory and generalization of the effective association number with co-solvent concentration is demonstrated feasible. Although several correlative models yield good results only for binary systems, this simplified cluster solvation model is applicable also for multi-component and co-solvent systems.

\section{List of symbols}

$A, B \quad$ parameter used in Eq. (23)

$a, b \quad$ parameters in equation of state 
$C_{1}, C_{2}$ parameters used in Eq. (31)

$f \quad$ fugacity

$G \quad$ Gibbs energy

$k \quad$ association number

$P \quad$ pressure

$R \quad$ universal gas constant

$T$ temperature

V volume

y solubility

$z \quad$ mole fraction base on chemical equilibrium

Greek letters

$\phi \quad$ fugacity coefficient

$\omega \quad$ acentric factor

\section{Subscripts}

c critical properties

eff effective value

F supercritical fluid

$i, j \quad$ component $i$ or $j$

$\mathrm{SF}_{k} \quad$ cluster

s solid

\section{Superscripts}

standard state

cal calculated value

exp experimental data

rxn reaction

sat saturated condition

\section{Acknowledgements}

The authors are grateful to the National Science Council, ROC, for supporting this research.

\section{References}

[1] A.S. Teja, C.A. Eckert, Ind. Eng. Chem. Res. 39 (2000) 4442-4444.

[2] M. Skerger, Z. Novk-Pintaric, Z. Kenz, Z. Kravanja, Fluid Phase Equilib. 203 (2002) 111-132.

[3] S. Garnier, E. Neau, P. Alessi, A. Cortesi, I. Kikic, Fluid Phase Equilib. 158-160 (1999) 491-500.

[4] C.C. Huang, M. Tang, W.H. Tao, Y.P. Chen, Fluid Phase Equilib. 179 (2001) 67-84.

[5] Y. Iwai, Y. Koga, T. Fukuda, Y. Arai, J. Chem. Eng. Jpn. 25 (1992) 757-760.

[6] J.S. Cheng, M. Tang, Y.P. Chen, Fluid Phase Equilib. 194-197 (2002) 483-491.

[7] J. Mendez-Santiago, A.S. Teja, Fluid Phase Equilib. 158-160 (1999) 501-510.

[8] J. Mendez-Santiago, A.S. Teja, Ind. Eng. Chem. Res. 39 (2000) 4767-4771. 
[9] O. Kajimoto, M. Futakami, T. Kobayashi, K. Yamasaki, J. Phys. Chem. 92 (1988) 1347-1352.

[10] T. Sasaki, H. Takeishi, Z. Yoshida, J. Supercrit. Fluids 15 (1999) 23-31.

[11] J. Otomo, S. Koda, Chem. Phys. 242 (1999) 241-252.

[12] C.J. Benmore, B.L. Tomberli, Ind. Eng. Chem. Res. 39 (2000) 4491-4495.

[13] B.L. Knuston, D.L. Tomasko, C.A. Eckert, P.G. Debenedetti, A.A. Chialvo, ACS Symposium Series 488, American Chemical Society, Washington, DC, 1992, Chapter 5.

[14] J.F. Brennecke, C.A. Eckert, AIChE J. 35 (1989) 1409-1427.

[15] M. Zhong, B. Han, J. Ke, H. Yan, D.Y. Peng, Fluid Phase Equilib. 146 (1998) 93-102.

[16] C. Jiang, Q. Pan, Z. Pan, J. Supercrit. Fluids 12 (1998) 1-9.

[17] D.Y. Peng, D.B. Robinson, Ind. Eng. Chem. Fundam. 15 (1976) 59-64.

[18] Y. Hu, E. Azevedo, D. Ludecke, J.M. Prauznitz, Fluid Phase Equilib. 17 (1984) 303-321.

[19] J. Hong, Y. Hu, Fluid Phase Equilib. 51 (1989) 37-51.

[20] R.M. Lemert, K.P. Johnston, Ind. Eng. Chem. Res. 30 (1991) 1222-1231.

[21] F.R. Lucien, N.R. Foster, J. Supercrit. Fluids 17 (2000) 111-134.

[22] M. Zhong, B. Han, H. Yan, D.Y. Peng, Fluid Phase Equilib. 134 (1997) 175-183.

[23] J. Chrastil, J. Phys. Chem. 86 (1982) 3016-3021.

[24] Y. Koga, Y. Iwai, Y. Hata, M. Yamamoto, Y. Aria, Fluid Phase Equilib. 125 (1996) 115-128.

[25] R.C. Reid, J.M. Prausnitz, B.E. Poling, The Properties of Gases and Liquids, fourth ed., McGraw-Hill, New York, 1987.

[26] A.L. McClellan, Tables of Experimental Dipole Moments, Freeman, San Francisco, 1963.

[27] T.E. Daubert, R.P. Danner, Physical and Thermodynamic Properties of Pure Chemicals: Data Compilation, Hemisphere Publishing Co., New York, 1989.

[28] P. Coutsikos, K. Magoulas, D. Tassios, J. Chem. Eng. Data 42 (1997) 463-466.

[29] D.R. Lide, H.P.R. Frederikse, CRC Handbook of Chemistry and Physics, 75th ed., CRC Press, Boca Raton, FL, 1994.

[30] D.S. Jan, F.N. Tsai, Ind. Eng. Chem. Res. 30 (1991) 1965-1970.

[31] E. Yu, M. Richter, P. Chen, X. Wang, Z. Zhang, L. Ind. Eng. Chem. Res. 34 (1995) 340-346.

[32] Y. Iwai, Y. Mori, N. Hosotani, H. Higashi, T. Furuya, Y. Arai, J. Chem. Eng. Data 38 (1993) 509-512.

[33] Y. Mori, T. Shimizu, Y. Iwai, Y. Arai, J. Chem. Eng. Data 37 (1992) 317-319.

[34] S. Sako, K. Shibata, K. Ohgaki, T. Katayama, J. Supercrit. Fluids 2 (1989) 3-8.

[35] D.H. Ziger, C.A. Eckert, Ind. Eng. Chem. Res., Process Des. Dev. 22 (1983) 582-588.

[36] A. Cortesi, I. Kikic, P. Alessi, G. Turtoi, S. Garnier, J. Supercrit. Fluids 14 (1999) 139-144.

[37] R.C. Weast (Ed.), CRC Handbook of Chemistry and Physics, 69th ed., CRC Press, Boca Raton, FL, 1989.

[38] M. Mukhopadhyay, G.V.R. Rao, Ind. Eng. Chem. Res. 32 (1993) 922-930.

[39] F.P. Lucien, N.R. Foster, Ind. Eng. Chem. Res. 35 (1996) 4686-4699.

[40] T. Bamberger, J.C. Erickson, C.L. Cooney, J. Chem. Eng. Data 33 (1988) 327-333.

[41] W.J. Schmitt, R.C. Reid, J. Chem. Eng. Data 31 (1986) 204-212.

[42] P.A. Wells, R.P. Chaplin, N.R. Foster, J. Supercrit. Fluids 3 (1990) 8-14.

[43] S.J. Macnaughton, N.R. Foster, Ind. Eng. Chem. Res. 33 (1994) 2757-2763.

[44] W.M. Cross, A. Akgerman, Ind. Eng. Chem. Res. 37 (1998) 1510-1518.

[45] S.S.T. Ting, S.J. Macnaughton, D.L. Tomasko, N.R. Foster, Ind. Eng. Chem. Res. 32 (1993) 1471-1481.

[46] M.D. Gordillo, M.A. Blanco, A. Molero, E.M. Ossa, J. Supercrit. Fluids 15 (1999) 183-190.

[47] W.J. Lyman, W.F. Reehl, D.H. Rosenblatt, Handbook of Chemical Property Estimate Method, American Chemical Society, Washington, DC, 1990.

[48] Y. Iwai, Y. Koga, H. Maruyama, Y. Arai, J. Chem. Eng. Data 38 (1993) 506-508.

[49] R.T. Kurnik, S.J. Holla, R.C. Reid, J. Chem. Eng. Data 26 (1981) 47-51.

[50] K.P. Johnston, D.H. Ziger, C.A. Eckert, Ind. Eng. Chem. Fundam. 21 (1982) 191-197.

[51] K.P. Johnston, C.A. Eckert, AIChE J. 27 (1981) 733-739.

[52] E. Kosal, G.D. Holder, J. Chem. Eng. Data 32 (1987) 148-150.

[53] M.A. McHugh, M.E. Paulaitis, J. Chem. Eng. Data 25 (1980) 326-329.

[54] L. Barna, J.M. Blanchard, E. Rauzy, C. Berro, J. Chem. Eng. Data 41 (1996) 1466-1469.

[55] K.D. Bartle, A.A. Clifford, S.A. Jafar, J. Chem. Eng. Data 35 (1990) 355-360.

[56] J. Ke, C. Mao, M.H. Zhong, B. Han, H. Yan, J. Supercrit. Fluids 9 (1996) 82-87.

[57] Y. Iwai, T. Fukuda, Y. Koga, Y. Arai, J. Chem. Eng. Data 36 (1991) 430-432. 
[58] S.T. Chung, K.S. Shing, Fluid Phase Equilib. 81 (1992) 321-341.

[59] R.A. Van Leer, M.E. Paulaities, J. Chem. Eng. Data 25 (1980) 257-259.

[60] J.M. Dobbs, J.M. Wong, R.J. Lahiere, K.P. Johnston, Ind. Eng. Chem. Res. 26 (1987) 56-65.

[61] F.P. Lucien, N.R. Foster, J. Chem. Eng. Data 43 (1998) 726-731.

[62] J.M. Dobbs, K.P. Johnston, Ind. Eng. Chem. Res. 26 (1987) 1476-1482.

[63] R.T. Kurnik, R.C. Reid, Fluid Phase Equilib. 8 (1982) 93-105.

[64] R.M. Lemert, K.P. Johnston, Fluid Phase Equilib. 59 (1990) 31-55.

[65] G.T. Liu, K. Nagahama, J. Supercrit. Fluids 9 (1996) 152-160.

[66] J.M. Dobbs, J.M. Wong, K.P. Johnston, J. Chem. Eng. Data 31 (1986) 303-308. 